\title{
Specific targeting of point mutations in EGFR L858R-positive lung cancer by CRISPR/Cas9
}

\author{
Alvin Ho-Kwan Cheung $\mathbb{1 0}^{1} \cdot$ Chit Chow ${ }^{1} \cdot$ Jinglin Zhang ${ }^{1,2,3} \cdot$ Yuhang Zhou ${ }^{1,2,3} \cdot$ Tingting Huang ${ }^{1,2,3,4}$. \\ Kayla Ching-Kei $\mathrm{Ng}^{1}$ • Terry Cho-Tsun $\mathrm{Or}^{1} \cdot$ Yoyo Yao Yao ${ }^{1}$ Yujuan Dong ${ }^{2,5}$ • Jackie Mei-Wah Fung ${ }^{1}$. Lei Xiong ${ }^{6}$. \\ Aden Ka-Yin Chan (1) ${ }^{1} \cdot$ Wai-Ming Raymond Lung ${ }^{1} \cdot$ Wei Kang ${ }^{1,2,3,4} \cdot$ Ka-Fai To ${ }^{1,2,3,4}$
}

Received: 1 December 2017 / Revised: 16 January 2018 / Accepted: 14 February 2018 / Published online: 10 May 2018

(c) United States \& Canadian Academy of Pathology 2018

\begin{abstract}
Cancer cells are defined genetically by the mutations they harbor, commonly single nucleotide substitutions. Therapeutic approaches which specifically target cancer cells by recognizing these defining genetic aberrations are expected to exhibit minimal side-effects. However, current protein-based targeted therapy is greatly limited by the range of genes that can be targeted, as well as by acquired resistance. We hypothesized that a therapeutic oligonucleotide-based strategy may address this need of specific cancer targeting. We used CRISPR/Cas9 system to target a commonly occurring EGFR point mutation, L858R, with an oligonucleotide guide that recognizes L858R as the suitable protospacer-adjacent motif (PAM) sequence for DNA cleavage. We found that this strategy, which utilized PAM to differentiate cancer mutation from normal, afforded high specificity to the extent of a single nucleotide substitution. The anti-L858R vehicle resulted in selective genome cleavage only in L858R mutant cells, as detected by Sanger sequencing and T7 Endonuclease I assay. Wild-type cells were unaffected by the same treatment. Digital PCR revealed 37.9 $\pm 8.57 \%$ of L858R gene copies were targeted in mutant. Only treated mutant cells, but not wild-type cells, showed reduction in EGFR expression and decreased cell proliferation. Treated mutant cells also formed smaller tumor load in vivo. This targeting approach is expected to be able to target a significant subset of the $15-35 \%$ cancer mutations with $\mathrm{C}>\mathrm{G}, \mathrm{A}>\mathrm{G}$, and $\mathrm{T}>\mathrm{G}$ point mutations. Thus, this strategy may serve as a useful approach to target cancer-defining mutations with specificity, to the extent of differentiating the change of a single nucleotide.
\end{abstract}

\section{Introduction}

Mutations are the defining features of cancer cells, which distinguish them from normal. The most common type of

Electronic supplementary material The online version of this article (https://doi.org/10.1038/s41374-018-0056-1) contains supplementary material, which is available to authorized users.

Wei Kang

weikang@cuhk.edu.hk

$\triangle$ Ka-Fai To

kfto@cuhk.edu.hk

1 Department of Anatomical and Cellular Pathology, State Key Laboratory of Oncology in South China, Prince of Wales Hospital, The Chinese University of Hong Kong, Hong Kong, China

2 Institute of Digestive Disease, Partner State Key Laboratory of Digestive Disease, The Chinese University of Hong Kong, mutations are single nucleotide substitutions, or point mutations. Although cancer-specific mutations are already being exploited to selectively target cancer cells, current-targeted therapy, being predominantly antibody based, has its limitations as many genes are thought to be not druggable at the protein level [1, 2]. A growing number of cancer mutations are found within the gene regulatory region, such as the enhancers [3] and promoters [4], such that changes in expression level of an essential cellular regulator are

Hong Kong, China

3 Li Ka Shing Institute of Health Science, Sir Y.K. Pao Cancer Center, The Chinese University of Hong Kong, Hong Kong, China

4 Shenzhen Research Institute, The Chinese University of Hong Kong, Shenzhen, China

5 Department of Surgery, The Chinese University of Hong Kong, Hong Kong, China

6 School of Biomedical Sciences, The Chinese University of Hong Kong, Hong Kong, China 
responsible for oncogenesis. Obviously, antibodies that target these regulators may not be effective, and indeed may be detrimental. In addition, tumor cells may acquire resistance to targeted therapy by additional mutations to the functional domain of the targeted gene, as illustrated by T790M mutation, which abolished response to first line tyrosine kinase inhibitors in EGFR mutation-positive lung cancer.

A drug that targets cancer genome by its defining mutation may be a solution to these problems. We hypothesized that an approach targeting cancer-defining mutation most likely requires an oligonucleotide using the Watson-Crick complementary base-pairing strategy [5]. Among current technologies, the CRISPR/Cas9 system appears to be a candidate. This system utilizes Cas9, a bacterial-derived nuclease, to cleave double-stranded DNA at a desired site by using an appropriately designed guide RNA (gRNA) complementary to the target sequence. For recognition by Cas9, the sequence NGG ( $\mathrm{N}=$ any nucleotide), termed "protospacer-adjacent motif," (PAM) is also required $3^{\prime}$ to the target site.

Cancer specificity requires perfect binding of an oligonucleotide to the target sequence, because the mutated gene in cancer may only differ from normal by one nucleotide, i.e., a point mutation. The CRISPR/Cas9 system has yet achieved perfect binding specificity between the gRNA and its target, and DNA cleavage still occurs if there is a nucleotide mismatch [6]. We hypothesized the PAM sequence may afford a better differentiating power.

We used a point mutation commonly encountered in lung cancer patients, L858R, as a model to test this hypothesis. L858R accounts for $42.5 \%$ of EGFR-mutated lung adenocarcinoma in Asians [7]. In this mutation, $\mathrm{T}-\mathrm{G}$ transversion occurs in exon 21 of $E G F R$, resulting in an activated kinase domain, and increased signal transduction to the downstream pro-survival pathways either partially or completely independent of receptor-binding ligands [8]. We designed an anti-L858R targeting vehicle, which incorporated the Cas9 nuclease and a L858R-specific gRNA. We expected the interesting finding that delivery of this same therapeutic vehicle would only selectively target the mutant but not the wild-type cells, in terms of cell proliferation.

\section{Materials and methods}

\section{Cell culture}

Mutant (NCI-H1975) and wild-type (786-O, NCI-H1650) cell lines with respect to the EGFR codon 858 locus were obtained from American Type Cell Culture, and was maintained in RPMI-1640 (Gibco, Grand Island, NY) supplemented with $10 \%$ fetal bovine serum (FBS) (Gibco), and placed in an incubator with a temperature of $37^{\circ} \mathrm{C}$, under $5 \% \mathrm{CO}_{2}$ humidity. 293TN cells (System biosciences, Palo Alto, CA), which was used for lentivirus packaging, was maintained in Dulbecco's Modified Eagle's Medium (DMEM) (Life Technologies, Carlsbad, CA) supplemented with $10 \%$ FBS under the same maintenance condition.

\section{DNA constructs and transduction}

The anti-L858R-targeting vehicle was assembled by cloning pL-CRISPR.EFS.GFP (Addgene \#57818), with the insert for positive control gRNA (5'-CACCGCAAGATCACAGATTTTGGGC-3'), anti-L858R gRNA (5'-CACCGTCAAGATCACAGATTTTGGG-3'), and negative control gRNA (5'-CACCGGTCAAGATCACAGATTTTGG-3'), using the restriction enzyme BsmBI (New England Biolabs, Ipswich, MA). Packaging of lentivirus was performed by co-transfecting the construct, pMD2.G (Addgene \#12259) and psPAX2 (Addgene \#12260) into 293TN cells, using FuGENE HD transfection reagent (Roche, Nutley, NJ). Lentivirus was collected at $48 \mathrm{~h}$ after transfection, and was subsequently used to infect the targeted cells, with the addition of Polybrene $(6 \mu \mathrm{g} / \mathrm{mL}$; Sigma-Aldrich, St Louis, $\mathrm{MO})$.

\section{Digital PCR}

Genomic DNA extracted from cancer cells was fragmented to $\sim 1500$ bp by the S220 Focused-ultrasonicator (Covaris, Woburn, MA). The concentration of the sonicated DNA samples was measured by Qubit 2.0 Fluorometer (Life Technologies). Parallel PCR amplification of wild type and L858R mutant sequences was performed using the forward primer 5'-GCAGCATGTCAAGATCACAGATT-3' and reverse primer $5^{\prime}$-CCTCCTTCTGCATGGTATTCTTTCT$3^{\prime}$. Probing of wild type and L858R mutant PCR amplicons were by the probes $5^{\prime}$-(VIC)AGTTTGGCCAGCCCAA (MGBNFQ)-3' and 5'-(FAM)AGTTTGGCCCGCCCAA (MGBNFQ)-3' (Life Technologies), where FAM is 6carboxyfluorescein and MGBNFQ is a minor groovebinding nonfluorescent quencher respectively. RainDance Source and Sense System (RainDance Technologies, Lexington, MA) was used for performing droplet digital PCR. Droplets containing single DNA template were emulsified and amplified in Bio-Rad C1000 thermal cycler with the reaction initiated at $95^{\circ} \mathrm{C}$ for $10 \mathrm{~min}$, followed by 50 cycles of $95^{\circ} \mathrm{C}$ for $15 \mathrm{~s}$ and $60^{\circ} \mathrm{C}$ for $1 \mathrm{~min}$ at a $0.5^{\circ} \mathrm{C} / \mathrm{min}$ ramp rate, and subsequently $98^{\circ} \mathrm{C}$ for $12 \mathrm{~min}$. End-point fluorescence measurement was performed with the RainDance Sense device (RainDance Technologies), and then analyzed with the RainDance Analyst Software (RainDance Technologies). Positive signals were defined where the $99 \%$ confidence interval of false positive (limit of blank) was exceeded, determined from assays with wild-type controls. 


\section{Surveyor assay}

Surveyor assay kit was purchased from Integrated DNA technologies (Coralville, IA). Briefly, genomic DNA extracted from transduced cancer cells was PCR amplified at the EGFR exon 21 locus with the forward primer 5'-ACGTTCGCCAGCCATAAGTC-3' and reverse primer 5'-AGCTCTGGCTCACACTACCA-3' followed by PCR product purification using a commercially available kit (Qiagen, Hilden, Germany). The PCR purification products were melted and reannealed in a thermal cycler according to the manufacturer's protocol, and then subsequently incubated with $1 \mu \mathrm{l}$ of Surveyor Nuclease $\mathrm{S}$ and $1 \mu \mathrm{l}$ of Enhancer $\mathrm{S}$ (Integrated DNA technologies) at $42^{\circ} \mathrm{C}$ for $1 \mathrm{~h}$. The digestion product was then subjected to electrophoresis with a $2 \%$ agarose gel.

\section{Western blot}

Cell lysis was performed in ice-cold radioimmunoprecipitation assay buffer (Cell Signaling Technology, Danvers, MA) containing phenoylmethylsulfonyl fluoride $(1 \mathrm{mmol} / \mathrm{L})$ and protease inhibitor. The lysate was centrifuged at $12,000 \times \mathrm{g}$ for $15 \mathrm{~min}$ to obtain the supernatant. The collected protein was suspended in sodium dodecyl sulfate buffer and resolved by sodium dodecyl sulfate-polyacrylamide gel electrophoresis. Transfer to polyvinylidene difluoride membranes (GE Healthcare, Piscataway, NJ) was carried out. The antibody against total EGFR and L858R-mutated EGFR were purchased from Cell Signaling Technology (Danvers, MA). The membranes were developed after probing with horseradish peroxidaseconjugated secondary antibodies.

\section{In vitro functional assays}

Cell proliferation was assessed using CellTiter 96 NonRadioactive Cell Proliferation Assay (MTT; Promega, Madison, WI) according to manufacturer's instruction. For colony formation assays in monolayer cultures, transduced cells were cultured for 10 days, and subsequently fixed with $70 \%$ ethanol for $15 \mathrm{~min}$ and stained with $2 \%$ crystal violet. Colonies with more than 50 cells per colony were counted.

\section{In vivo tumor formation assay}

The protocol of in vivo tumorigenicity model was described previously [9]. Tumor was allowed to grow for 3 weeks, then the mice were sacrificed. The xenografts were taken out for evaluation. Average weights of mice for each group were defined as center values and standard deviations were used for error bars. The animal experimental procedures were approved by Department of Health, Hong Kong.

\section{Statistical analysis}

Continuous variables were expressed as means \pm SD. Treatment groups were compared with the independent or paired sample $t$-test where appropriate. $p$-values $<0.05$ were considered statistically significant. Analyses were performed with the SPSS statistics software (version 20, SPSS Inc., Chicago, IL).

\section{Results}

\section{Strategies for selective targeting of point mutations}

EGFR-exon 21 L858R mutant cancer cells and wild-type control cells were transduced with the same plasmid carrying the Cas9 protein and gRNA specifically designed to target the mutant DNA (Fig. 1a). The binding site of this anti-L858R gRNA is placed immediately upstream to the site of PAM, where the mutation L858R lies. With this mutation, the requirement of PAM for DNA cleavage is thus only fulfilled in mutant cells but not wild-type cells. As no effect is to be expected with this anti-L858R gRNA in the wild-type cells, another gRNA, which was designed to bind one base-pair downstream to the binding site of antiL858R gRNA, served as a positive control. This positive control gRNA could cleave both wild-type and mutant DNA, because of the presence of PAM sequence GGG in the mutant cells and TGG in the wild-type cells. On the other hand, the negative control gRNA bound to one basepair upstream to the binding site of anti-L858R gRNA and could not cleave DNA due to the lack of an appropriate PAM. Further selection of positively transduced cells was not performed in order to emphasize the translational and therapeutic relevance of this approach. It was ascertained that the transduction efficiencies of the anti-L858R targeting vehicle to both mutant and wild-type cells were similar (Fig. 1b).

\section{DNA cleavage occurs in mutant but not wild-type cells}

As a result of double-stranded DNA cleavage by CRISPR/ Cas9, the targeted cells undergo DNA repair principally by virtue of nonhomologous end-joining (NHEJ). However, this process is imperfect in that insertions-deletions (indels) are often introduced at the cleavage site at the same time [10]. The detection of these indels is evidence of successful genome cleavage. By Sanger sequencing, genome cleavage was present only in mutant cells treated with the anti-L858R targeting vehicle but not in wild-type cells, as evident by the occurrence of frameshift mutation at the cleavage site in mutant cells only (Fig. 2a). 

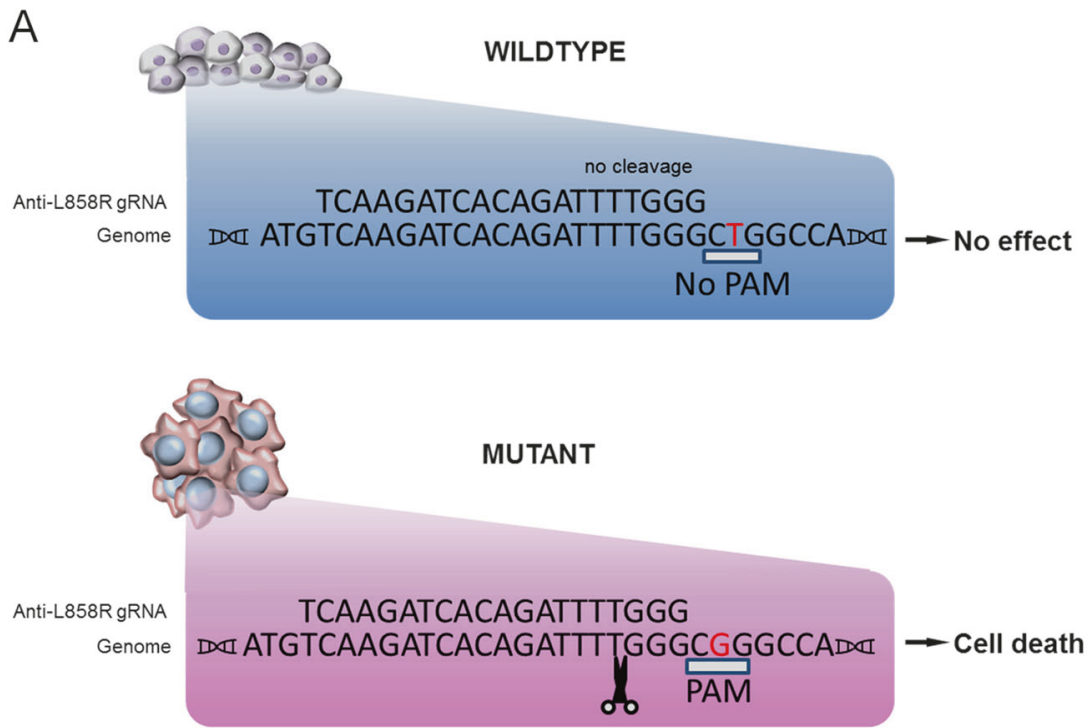

B

Mutant

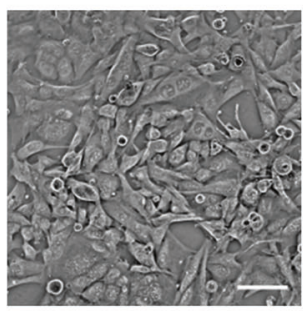

GFP
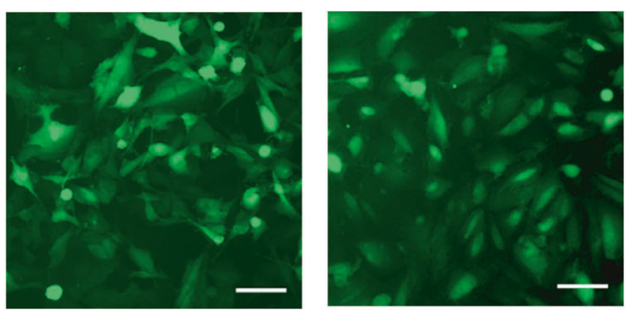

Wildtype 786-0
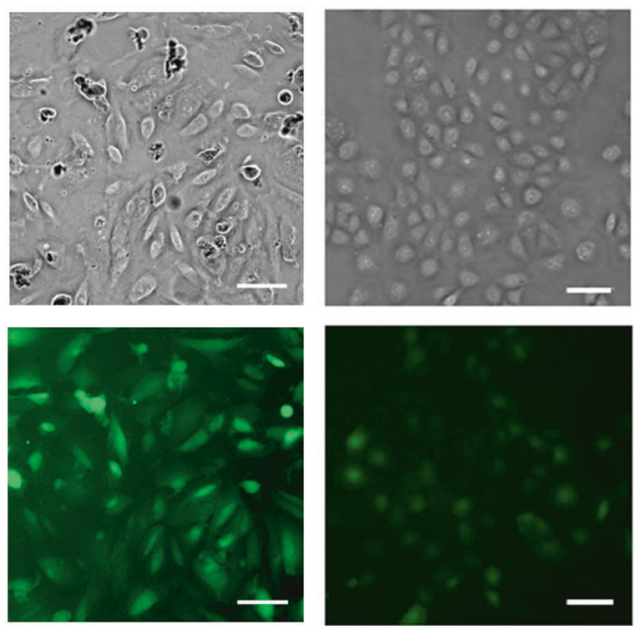

Fig. 1 Selective mutant cancer cell targeting by using CRISPR/ Cas9 system as a targeting approach. a Schematic diagram illustrating the targeting strategy. The CRISPR/Cas9 system utilizes Cas9 nuclease to cleave double-stranded DNA at a targeted site by binding of a guide RNA (gRNA) to the target sequence, with the prerequisite of the sequence NGG $(\mathrm{N}=$ any nucleotide), termed protospacer-adjacent motif (PAM), at immediate $3^{\prime}$ of the target site. When a point mutation in cancer changes the DNA sequence to NGG, such as in the T-G transversion of L858R (shown red), the requirement of PAM for DNA cleavage is only fulfilled in mutant cells, but not wild-type cells. This results in cleavage of genome specifically in the mutant cells, leading to cell death due to EGFR downregulation. For clarity, the reversecomplementary sequence of the L858R targeting gRNA is shown. The binding sites of positive control gRNA (5'-CAAGATCACAGATTTTGGGC) and negative control gRNA (5'-GTCAAGATCACAGATTTTGG) are in the close proximity to the binding site of antiL858R gRNA. b Transduction of the anti-L858R targeting vehicle. The anti-L858R-targeting vehicle showed similar efficiency when transduced to mutant (NCI-H1975) and wild-type cells, as visualized by green fluorescent protein (GFP). Scale bar, $100 \mu \mathrm{m}$
To further validate the absence of genome cleavage in wild-type cells, a more sensitive assay using $\mathrm{T} 7$ endonuclease I to detect DNA mismatch was performed. The test is positive when DNA sequences of any two alleles are different in $>1-5 \%$ [11], as can be expected when indels were generated randomly in either chromosomes in CRISPRtargeted cells. The mutant cells, being heterozygous at the L858R locus, served as the positive control for the assay. No genome cleavage was detected in anti-L858R vehicle- treated wild-type cells (Fig. 2b). On the other hand, to further examine the genome targeting efficiency in mutant cells, digital PCR was carried out. It was demonstrated that $1.14 \pm 0.24$ out of 3.02 copies of L858R gene was targeted on average in mutant cells ( $p<0.0005$, Fig. $2 c)$. It must be taken into account that although transduction efficiency was high (Fig. 1b), CRISPR/Cas9-mediated DNA cleavage only occurs in some proportion of the transduced cells [11, 12]. With the current finding, it was likely that DNA cleavage 
A

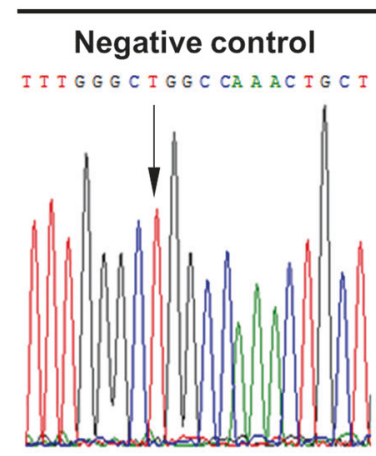

WILDTYPE

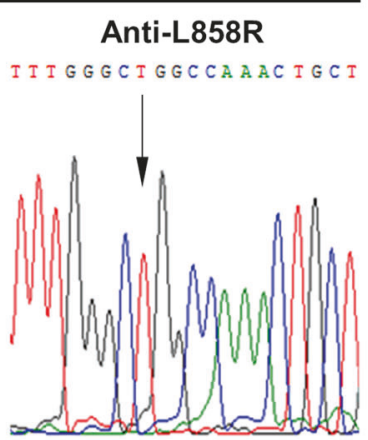

MUTANT

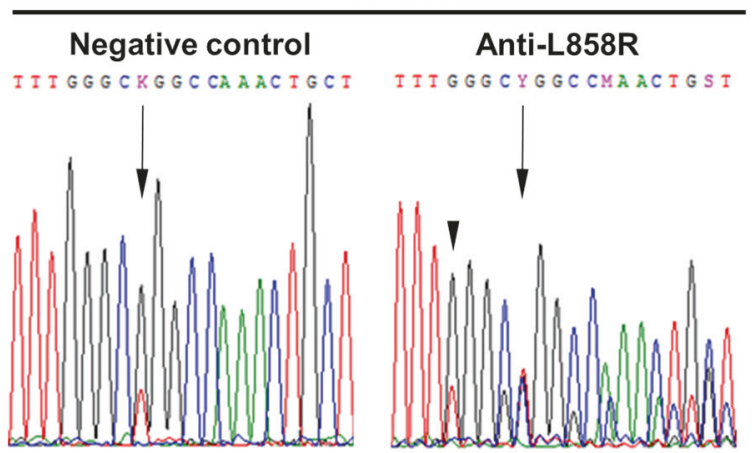

B

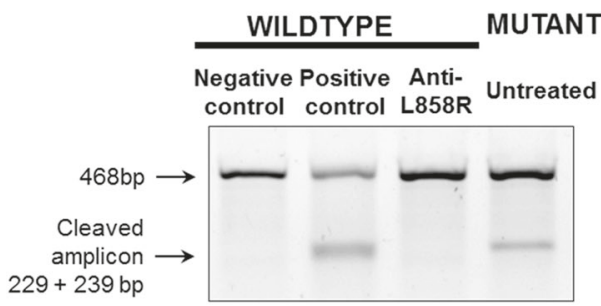
C MUTANT

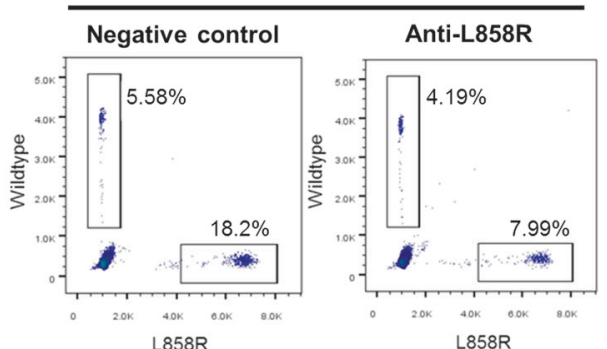

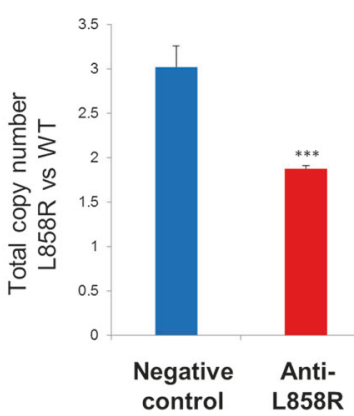

Fig. 2 Genome targeting occurred in L858R mutant cells but not wild-type cells. a By Sanger sequencing of PCR products, occurrence of frameshift mutation in mutant cells (NCI-H1975) treated with the anti-L858R targeting vehicle was detected. Note the mutant cells harbor T $-\mathrm{G}$ transversion (arrows) with amplification of the mutant allele. Genome cleavage by Cas 9 occurs 3-4 bases upstream to PAM, and frameshift mutation occurred as indel was introduced at the cleavage site (arrowhead). Wild-type cells (786-O) showed no such effect. b In wild-type cells, cleavage of the EGFR-amplified PCR amplicons ( $468 \mathrm{bp}$ ) by Surveyor assay occurs if genome targeting has taken place. Genomic DNA of the mutant cells served as a positive control for the assay. c Digital PCR detecting for L858R and the wild-type alleles in mutant cells, and showed decrease in copies of L858R alleles in targeted cells. $* * * p<0.0005$, two-tailed $t$-test

had occurred in at least $37.9 \pm 8.57 \%$ of the cancer cells. Also, because we expected decreased proliferation or death in cells where L858R was completely cleaved, this population could be underrepresented in the assay and thus the cleavage efficiency was underestimated.

\section{Functional effects of DNA cleavage only occur in mutant cells}

Functionally, the anti-L858R targeting vehicle demonstrated exquisite specificity for mutant cells. EGFR protein expression was downregulated in mutant only (Fig. 3a). Mutant cells treated with the anti-L858R targeting vehicle exhibited a decrease in proliferation by MTT assay $(p<$ 0.0005 , Fig. $3 b)$ and colony formation assay $(p<0.0005$, Fig. 3c), while no such inhibitory effect was observed in the wild-type cells. The results were consistent with the role of driver mutation $\mathrm{L} 858 \mathrm{R}$ in promoting tumor proliferation in cancer cells, with consequences of CRISPR/Cas9-mediated knockout demonstrated at the DNA, protein, and functional levels only in the mutant cells. On the other hand, the specificity of this system entails that the wild-type cells were unaffected at any of these levels.

\section{In vivo tumor cell suppression with targeting vehicle}

To evaluate the concept of specific point mutation targeting as a potential therapeutic technique, we further investigated the effect of anti-L858R targeting vehicle on tumor cells in vivo. In mice, anti-L858R treated cancer cells formed smaller tumors, with weight of $0.062 \pm 0.038 \mathrm{~g}$, versus $0.126 \pm 0.026 \mathrm{~g}$ for tumors formed by control cells $(p=$ 0.015 , Fig. $3 \mathrm{~d}$ ). This demonstrated that significant reduction in tumor burden may be possible in the in vivo setting when delivery of anti-L858R targeting vehicle to cancer cells is achieved, underpinning the translational potential for this strategy.

\section{Discussion}

Current targeted therapy is limited by the range of targetable genes because it is predominantly antibody based. To overcome this obstacle, newer approaches, such as the application of synthetic lethality, have emerged $[2,13]$. This approach targets genes which are lethal to the cell only 


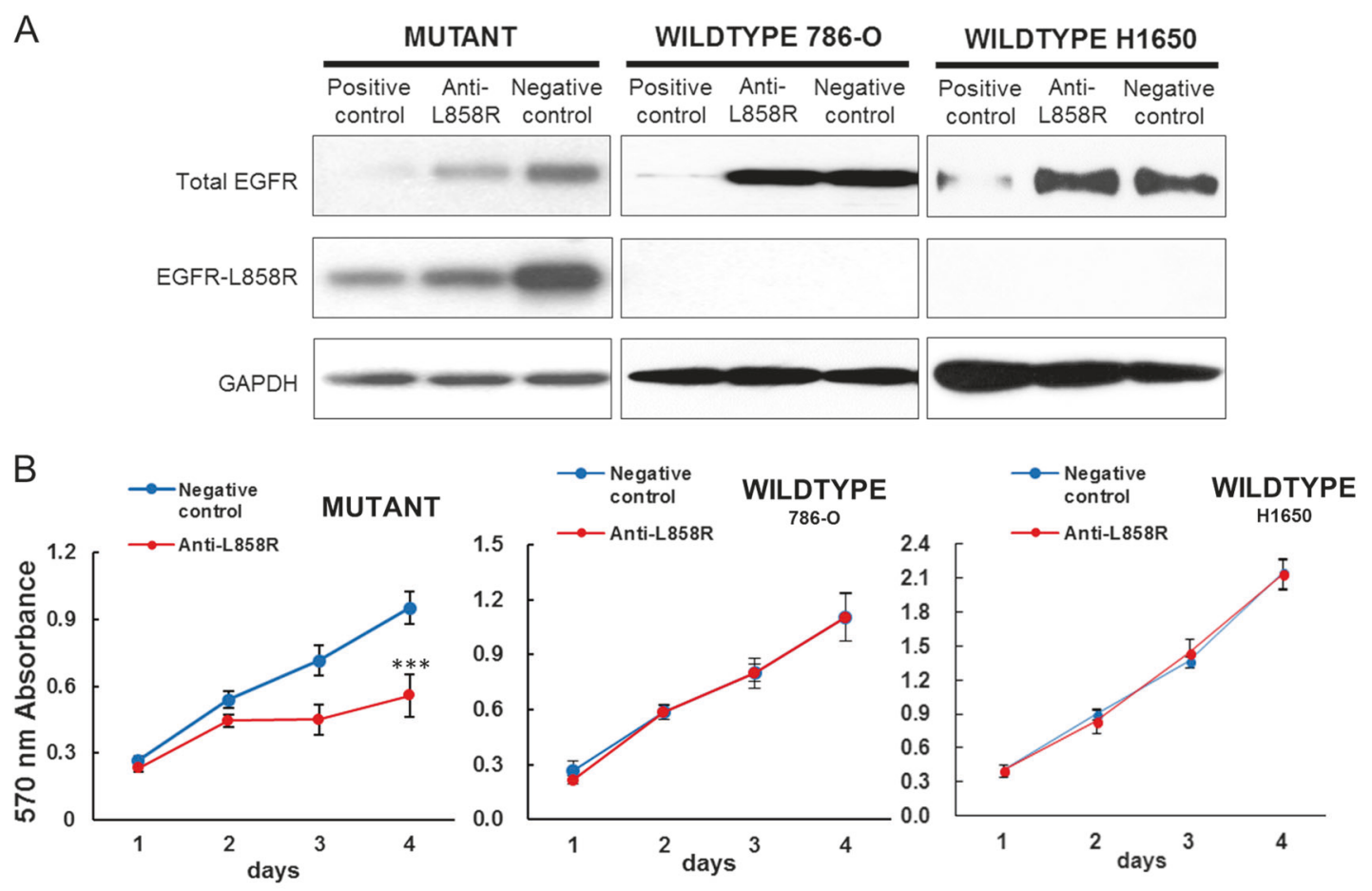

C



D


when there is an addictive effect to the cancer-driving oncogenes. Although promising, this approach still suffers from some degree of non-specificity, as genes in the normal cells will also be disrupted, only not to the extent of lethality [2]. This approach currently relies strongly on disrupting DNA damage repair machinery and can pose hazards to the 
Fig. 3 Functional studies demonstrated decreased EGFR expression and cell proliferation in L858R mutant cells but not wild-type cells with the anti-L858R targeting vehicle. a Western blot analyses of total EGFR and L858R-mutated EGFR expression level. b MTT and c colony formation assays of wild-type and mutant cells treated with the anti-L858R targeting vehicle, revealing inhibitory effects only on mutant but not wild-type cells. $* * * p<0.0005$, two-tailed $t$-test. $\mathbf{d}$ In vivo tumor formation with mutant cells treated with anti-L858R (red circle) targeting vehicle resulted in decreased tumor weight compared to those treated with negative control (blue circle). ${ }^{*} p=0.015$, twotailed $t$-test

normal cells. To afford cancer cell-specific targeting, a therapeutic approach acting on the cancer-defining mutation at the genome level may prove useful, particularly when resistance to targeted therapy has developed.

There are six different types of point mutations. With the most widely used Streptococcus pyogenes derived Cas9 enzyme for CRISPR/Cas9 system, it is apparent that the targetable types of mutation, as well as the mutation context are restricted by the PAM sequence $\mathrm{NGG}(\mathrm{N}=$ any nucleotide). With these constraints, $\mathrm{C}>\mathrm{G} / \mathrm{G}>\mathrm{C}, \mathrm{A}>\mathrm{G} / \mathrm{T}>$ $\mathrm{C}$, and $\mathrm{T}>\mathrm{G} / \mathrm{A}>\mathrm{C}$ are valid targets (Supplementary figure 1). In the common cancer types, these types of mutations together can account for 15-35\% of all mutations [14]. $\mathrm{C}>\mathrm{G} / \mathrm{G}>\mathrm{C}$ can occur commonly as a result of cytidine deaminase activity, which converts cytosine to uracil, and subsequent removal of uracil generates an abasic site [15]. A $>$ G/T > C mutation predominantly occurs in breast, stomach, and uterine cancers and is associated with defective DNA mismatch repair [15]. Where the mutation context is considered, the targeted site requires the presence of guanine at immediate upstream or immediate downstream (Supplementary figure 1). The proportion of cancer in which this mutation context can be met varies widely among cancer types. For example, for $\mathrm{T}>\mathrm{G}$ mutations in lung cancers, up to $41 \%$ has a downstream guanine, while for colorectal cancers, only $25 \%$ of all $\mathrm{T}>\mathrm{G}$ mutation has a downstream guanine [14]. Needless to say, the everexpanding repertoire of variants of Cas9 enzymes with different PAM specificity is encouraging to this therapeutic approach [16]. Worth mentioning are the Neisseria meningitidis derived Cas9, and the Francisella novicida derived Cpf1 nuclease, which feature PAM sequences that contain thymine [17]. These nucleases potentially provide the opportunity that the remaining types of point mutations, i.e., $\mathrm{C}>\mathrm{T} / \mathrm{G}>\mathrm{A}, \mathrm{G}>\mathrm{T} / \mathrm{C}>\mathrm{A}$, and $\mathrm{A}>\mathrm{T} / \mathrm{T}>\mathrm{A}$, can be targeted. The common $\mathrm{C}>\mathrm{T}$ mutation is often associated with spontaneous deamination of methylcytosine, defective DNA mismatch repair, and ultraviolet radiation [15].

Although the current study applies a straightforward approach of knocking out an oncogene by NHEJ, this does not imply that only oncogenes may be targeted by the CRISPR/Cas9 system. This system can potentially correct mutated nucleotide by means of homology directed repair (HDR), which mediates repair of double-strand break by means of recombination with an exogenous DNA template. HDR is possible only in dividing cells but not nondividing cells [18], an apparent advantage to cancer targeting, but it is hindered by its much lower rate of occurrence compared to NHEJ [19]. Nevertheless, developments are in progress to improve HDR efficiency in vitro $[19,20]$ and in vivo [18]. An innovative recent study targeted cancer cell by means of inserting an exogenous herpes virus type 1 thymidine kinase gene at the abnormal chromosomal rearrangement site, making the cell susceptible to ganciclovir as if it was a herpes virus infected cell [21]. Chromosomal rearrangements in cancer cells provide a specific target for CRISPR/Cas9 because the stretch of DNA sequences to which the gRNA binds is completely absent in normal cells. Strategies such as that proposed in the current study which target point mutations, a more common aberration found in cancer, are important to be further explored.

The current targeting efficiency of $37.9 \%$ was constrained by two major parameters, including the transduction efficiency of the vehicle and subsequently the cleavage efficiency of the protein Cas9. The former appeared to be a less significant factor in this study, given that expression of the vehicle was demonstrated in the vast majority of cells (Fig. 1b). For the latter, the efficiency of Cas9 cleavage and subsequent NHEJ is typically reported to average at about $40 \%$ [19, 22]. This efficiency largely depends on the sequence of the targeted site [23], which in turn relates to the sequence of the gRNA [24]. It may be necessary to administer repeated dose of the targeting vehicle for a sustained tumor suppressive effect. Importantly, unlike protein-based targeted therapy, because this approach targets the fundamental genetic change harbored by the tumor cells, it is less likely that acquired resistance may be developed by clonal selection of tumor cells, which contain additional genetic aberrations of the same gene.

Indels generated by genome cleavage by Cas9 cause frameshift mutations in about two-thirds of the targeted alleles, and in-frame mutations in the remaining one-third [25]. It was demonstrated that both types of mutations, when occurring in a functional domain, are as effective in abolishing the gene function, by leading to an unstable or nonfunctional protein $[25,26]$. Frameshift mutations can also result in premature termination codons, which result in nonsense-mediated mRNA decay [26]. Although random in nature, it is usually not a concern that these indels may lead to a deleterious mutation that promotes an undesirable modification of gene function or gain of gene expression. Only in rare situations may this be an issue, and one scenario is when translation is maintained by the presence of an alternative start codon [27]. This occurs when the CRISPRtargeted site is placed too upstream at the $5^{\prime}$ exon, such that 
a near-full stretch of normal protein sequence can still be translated by using an alternate initiation codon $3^{\prime}$ to the targeted site, effectively bypassing the CRISPR generated indel $[27,28]$. Protein expression will then be unaltered. This underpins the importance of careful gRNA design in a therapeutic setting, in that the target sequence should preferably direct to a functional domain of the cancer-driving gene, such as the tyrosine kinase domain of EGFR in the current study.

A limitation of this study is that the extent of off-target cleavage remains undetermined, although adverse phenotypic changes were not detected in control cells. Because off-target cleavage profile is different for every gRNA, depending on its sequence, each gRNA should be individually tested before clinically used. As algorithms to predict off-target sites are still imperfect, the determination of offtarget binding of individual gRNAs will likely require the application of next generation sequencing. Partially solving the problem is the engineering of the Cas9 nuclease into a "nickase," which creates single-strand breaks rather than double-strand breaks [29]. Genome targeting with a dual nickase system has been shown to achieve better target specificity [29]. As similar to other novel treatments, the successful clinical application of CRISPR/Cas9 must require a careful assessment on its benefit to risk ratio in different situations, be it for treating infections, cancers, or genetic diseases. The efficacy of existing drugs must for certain be put into consideration. The prognostically guarded cancer patients are in need for more treatment options.

Although PAM can be a nuisance to researchers because it greatly restricts the editable genome, on the other hand it provides unparalleled specificity that the gRNA sequence cannot confer. Targeting cancer genome allows the attractive possibility of targeting only the mutant allele of an essential cell regulator, as well as mutations in the noncoding regions, such as the promoters or enhancer elements. Translational studies using this approach can be expected to be highly feasible, with the recent developments of vectors, which are more robust to be packed into virus used in vivo [30-32], and the advent in nanoparticle delivery technology [31]. As it is the trend to interrogate patients' genome for cancer treatment, specific targeting of cancer-defining mutations appears to be the logical step ahead for precision medicine.

Acknowledgements The technical assistance from Dr. Tom Pok-Man Hau of the Core Facility of Chinese University of Hong Kong, Sir Y. K. Pao Cancer Center, and Dr. Wendy Law of the molecular diagnostic team of Department of Anatomical of Cellular Pathology, Chinese University of Hong Kong, are gratefully appreciated.

\section{Compliance with ethical standards}

Conflict of interest The authors declare that they have no conflict of interest.

\section{References}

1. Verdine GL, Walensky LD. The challenge of drugging undruggable targets in cancer: lessons learned from targeting BCL-2 family members. Clin Cancer Res. 2007;13:7264-70.

2. McLornan DP, List A, Mufti GJ. Applying synthetic lethality for the selective targeting of cancer. N Engl J Med. 2014;371: 1725-35.

3. Mansour MR, Abraham BJ, Anders L, et al. An oncogenic superenhancer formed through somatic mutation of a noncoding intergenic element. Science. 2014;346:1373-7.

4. Huang FW, Hodis E, Xu MJ, et al. Highly recurrent TERT promoter mutations in human melanoma. Science. 2013;339:957-9.

5. Levin AA. Targeting therapeutic oligonucleotides. N Engl J Med. 2017;376:86-8.

6. Fu Y, Foden JA, Khayter C, et al. High-frequency off-target mutagenesis induced by CRISPR-Cas nucleases in human cells. Nat Biotechnol. 2013;31:822-6.

7. Mok TS, Wu Y-L, Thongprasert S, et al. Gefitinib or carboplatin-paclitaxel in pulmonary adenocarcinoma. New Engl J Med. 2009;361:947-57.

8. Sharma SV, Bell DW, Settleman J, Haber DA. Epidermal growth factor receptor mutations in lung cancer. Nat Rev Cancer. 2007;7:169-81.

9. Huang T, Kang W, Zhang B, et al. miR-508-3p concordantly silences NFKB1 and RELA to inactivate canonical NF- $\mathrm{KB}$ signaling in gastric carcinogenesis. Mol Cancer. 2016;15:9.

10. Cong L, Ran FA, Cox D, et al. Multiplex genome engineering using CRISPR/Cas systems. Science. 2013;339:819-23.

11. Ousterout DG, Kabadi AM, Thakore PI, et al. Multiplex CRISPR/ Cas9-based genome editing for correction of dystrophin mutations that cause Duchenne muscular dystrophy. Nat Comm. 2015;6: 6244.

12. Mali P, Yang L, Esvelt KM, et al. RNA-guided human genome engineering via Cas9. Science. 2013;339:823-6.

13. Cox AD, Fesik SW, Kimmelman AC, Luo J, Der CJ. Drugging the undruggable RAS: mission possible? Nat Rev Drug Discov. 2014;13:828-51.

14. Kandoth C, McLellan MD, Vandin F, et al. Mutational landscape and significance across 12 major cancer types. Nature. 2013;502: 333-9.

15. Alexandrov LB, Nik-Zainal S, Wedge DC, et al. Signatures of mutational processes in human cancer. Nature. 2013;500:415-21.

16. Kleinstiver BP, Prew MS, Tsai SQ, et al. Engineered CRISPRCas9 nucleases with altered PAM specificities. Nature. 2015;523:481-5.

17. Lee CM, Cradick TJ, Bao G. The Neisseria meningitidis CRISPRCas9 system enables specific genome editing in mammalian cells. Mol Ther. 2016;24:645-54.

18. Suzuki K, Tsunekawa Y, Hernandez-Benitez R, et al. In vivo genome editing via CRISPR/Cas9 mediated homologyindependent targeted integration. Nature. 2016;540:144-9.

19. Maruyama T, Dougan SK, Truttmann MC, et al. Increasing the efficiency of precise genome editing with CRISPR-Cas9 by inhibition of nonhomologous end joining. Nat Biotechnol. 2015;33:538-42.

20. Renaud J-B, Boix C, Charpentier M, et al. Improved genome editing efficiency and flexibility using modified oligonucleotides with TALEN and CRISPR-Cas9 nucleases. Cell REp. 2016;14:2263-72.

21. Chen Z-H, Yan PY, Zuo Z-H, et al. Targeting genomic rearrangements in tumor cells through Cas9-mediated insertion of a suicide gene. Nat Biotechnol. 2017;35:543-50.

22. Wang H, Yang H, Shivalila CS, et al. One-step generation of mice carrying mutations in multiple genes by CRISPR/Cas-mediated genome engineering. Cell. 2013;153:910-8. 
23. Liu X, Homma A, Sayadi J, et al. Sequence features associated with the cleavage efficiency of CRISPR/Cas9 system. Sci Rep. 2016;6:19675.

24. Doench JG, Hartenian E, Graham DB, et al. Rational design of highly active sgRNAs for CRISPR-Cas9-mediated gene inactivation. Nat Biotechnol. 2014;32:1262-7.

25. Shi J, Wang E, Milazzo JP, et al. Discovery of cancer drug targets by CRISPR-Cas9 screening of protein domains. Nat Biotechnol. 2015;33:661-7.

26. Popp MW, Maquat LE. Leveraging rules of nonsense-mediated mRNA decay for genome engineering and personalized medicine. Cell. 2016;165:1319-22.

27. Makino S, Fukumura R, Gondo Y. Illegitimate translation causes unexpected gene expression from on-target out-of-frame alleles created by CRISPR-Cas9. Sci Rep. 2016;6:39608.
28. Neu-Yilik G, Amthor B, Gehring NH, et al. Mechanism of escape from nonsense-mediated mRNA decay of human $\beta$-globin transcripts with nonsense mutations in the first exon. RNA. 2011;17: 843-54.

29. Kim E, Kim S, Kim DH, et al. Precision genome engineering with programmable DNA-nicking enzymes. Genome Res. 2012;22: 1327-33.

30. Swiech L, Heidenreich M, Banerjee A, et al. In vivo interrogation of gene function in the mammalian brain using CRISPR-Cas9. Nat Biotechnol. 2015;33:102-6.

31. Yin H, Song CQ, Dorkin JR, et al. Therapeutic genome editing by combined viral and non-viral delivery of CRISPR system components in vivo. Nat Biotechnol. 2016;34:328-33.

32. Kay MA. State-of-the-art gene-based therapies: the road ahead. Nat Rev Genet. 2011;12:316-28. 